\title{
Opinion Control in a Team with Complete and Incomplete Communication ${ }^{\star}$
}

\author{
Vladimir V. Mazalov ${ }^{1,3}$, Yulia A. Dorofeeva ${ }^{1,2}$ and Elena M. Parilina ${ }^{3}$ \\ 1 Institute of Applied Mathematical Research, \\ Karelian Research Center of the Russian Academy of Sciences, \\ 11, Pushkinskaya str., Petrozavodsk 185910, Russia \\ E-mail: vmazalov@krc.karelia.ru \\ 2 Petrozavodsk State University, Russia, Petozavodsk, \\ 185910, Lenina pr., 33 \\ E-mail: julana2008@yandex.ru \\ 3 Saint Petersburg State University, \\ 7/9 Universitetskaya nab., Saint Petersburg 199034, Russia \\ E-mail: e.parilina@spbu.ru
}

\begin{abstract}
We examine the influence of management of the opinion of a group members, while the group of agents are considered with different communication structures. We consider the optimization problem in dynamics with feedback information structure. In the model, the communication structure plays an important role since not all agents are directly connected with each other. The influence of the agents on each other is taken into account in the equation of dynamics of agents' opinions. An agent weights the other agents' opinions and after that forms the next step opinion. Two types of communication structures are considered in the model. The results of numerical modeling illustrate the influence of some parameters of the model (control, influence power of agents on each other) on the optimal opinion dynamics.
\end{abstract}

Keywords: opinion dynamics, average opinion, feedback optimal control, Bellman function, communication structure.

\section{Introduction}

Decision making often takes place as a result of consensus reached after negotiations. De Groot model (De Groot, 1974) is a pioneer work in this area and one of the simplest models used in modeling negotiations initiated to reach a consensus. In this model, the opinion of the participants changes during the negotiations, depending on their "degree of trust" to each other. And a consensus is reached if there is a limit matrix of influence. The formation of public opinion under the influence of various social factors is described in the Friedkin-Johnsen model (Friedkin and Johnsen, 1990), which is an analogue of the De Groot model. As the authors emphasize, this pattern establishes the pattern of interaction in large and small-sized social communities.

In the dynamics of Hegselmann-Krause (Hegselmann and Krause, 2005), participants exchange opinions only with those who are the part of their "circle of communication". The size of this "circle" can be changed, so we can examine the large groups of agents, as well as the small communities. In this case, reaching a

\footnotetext{
* The work of the first and third authors was supported by the Russian Science Foundation under grant no. 17-11-01079.
} 
consensus is directly related to the number of subgroups into which the whole group of agents is divided, and the opinions are changed due to "averaging". Reaching a consensus in a group with centers of influence is described in (Bauso et al., 2016; Bure et al., 2017). We should also notice the models (Weisbuch et al., 2005; Sirbu et al., 2016). In the mentioned papers, there is no participant who controls the opinions of other agents, and all agents are symmetric. In (Mazalov and Parilina, 2020), the problem of control of the agents' opinions via influence on some agents of a social network is examined. In this setting, players control the agents' opinions. The purpose of control is to make the average opinion of agents as closer as possible to the desired one. Modeling of the dynamic process is made for a graph represented society. The graph communication structure influences the cooperation structure as well (see Parilina and Sedakov, 2014). The models on opinion controls with feedback information structure are examined in (Sedakov and Zhen, 2019; Rogov and Sedakov, 2020; Dorofeeva, 2020).

However, in most papers the communication structure of the agents has not taken into account. This factor is important, since the presence or absence of communication between community members directly affects the process of forming opinions in their iterations. The paper is devoted to the opinion dynamics taking into account the structure of interactions between the agents, as well as assessing the influence of control on the finally formed opinions.

The paper is organized as follows. Section 2 contains a general description of the model without specifying communication structures. In Section 3 we introduce a model with a communication structure given by a complete graph and find the optimal control for a given problem. In Section 4 we examine the model with incomplete communication structure in which one link is deleted from complete structure. Numerical simulations demonstrating the results of the previous sections are given in Section 5. We briefly conclude in Section 6.

\section{Model}

We consider the opinion dynamics in the society with an infinite time horizon. A society is represented by a pair of $(N, g)$, where $N$ is a finite set of society agents, and $g$ is a graph that reflects the communication structure of the society. Graph $g=(N, E)$ is defined by $N$, the set of vertices, and $E$, the set of edges. Besides the society agents, there is an independent member of the society, called player, who controls the opinion of the agents. As an example, we can consider the media center as a player influencing the agents' opinions. The dynamics of opinions in the model is given by

$$
x_{i}(t+1)=x_{i}(t)+a_{i}\left(\frac{x_{i}(t)+\sum_{j \in S_{i}} x_{j}(t)}{\left|S_{i}\right|+1}-x_{i}(t)\right)+u_{i}(t), i \in N \text {, }
$$

where $x_{i}(t) \in \mathbb{R}^{1}$ is an opinion of agent $i$ at time $t, S_{i}=\{j:(i, j) \in E\}$ is the set of neighbors of agent $i$ in graph $g, a_{i} \in \mathbb{R}_{+}$is a coefficient defined for any society agent, $u_{i} \in U \subset[0, \infty)$ is a control of the player on agent $i$. A player can influence the subset of agents. The choice of agents to be influenced is an important task which is not examined in the paper.

The player's goal is to maintain the opinion of society agents closer to a certain level $\hat{x}$ minimizing their costs on control the agents' opinion. The following 
functional represents the player's costs:

$$
J(u)=\sum_{t=0}^{\infty} \delta^{t} \sum_{i=1}^{n}\left[\left(x_{i}(t)-\hat{x}\right)^{2}+\gamma u_{i}^{2}(t)\right],
$$

where $\delta \in(0 ; 1)$ is a discount factor, $\gamma>0$ is the cost per unit control.

In the next sections, we will examine the case of three agents and two types of communication structure represented by a complete and incomplete graphs. We also assume that the player can influence the opinion of a unique agent in the society.

\section{Model with Communication Structure Given by a Complete Graph}

Consider a society defined by three agents who communicate with each other via structure represented by a graph on Fig. 1 .

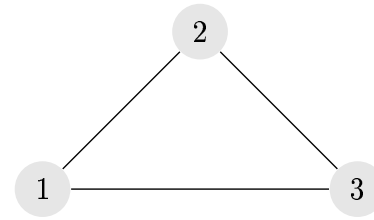

Fig. 1. Communication structure represented by a complete graph.

The dynamics of agents' opinion (state variable) is defined by the following equations:

$$
\begin{aligned}
& x_{1}(t+1)=x_{1}(t)+a_{1}\left(\frac{x_{1}(t)+x_{2}(t)+x_{3}(t)}{3}-x_{1}(t)\right), \\
& x_{2}(t+1)=x_{2}(t)+a_{2}\left(\frac{x_{1}(t)+x_{2}(t)+x_{3}(t)}{3}-x_{2}(t)\right)+u(t), \\
& x_{3}(t+1)=x_{3}(t)+a_{3}\left(\frac{x_{1}(t)+x_{2}(t)+x_{3}(t)}{3}-x_{3}(t)\right),
\end{aligned}
$$

or in vector form:

$$
x(t+1)=A x(t)+B u(t),
$$

where $x(t)=\left(x_{1}(t), x_{2}(t), x_{3}(t)\right)^{\prime} \in \mathbb{R}^{3}$, and

$$
A=\left(\begin{array}{ccc}
1-\frac{2 a_{1}}{3} & \frac{a_{1}}{3} & \frac{a_{1}}{3} \\
\frac{a_{2}}{3} & 1-\frac{2 a_{2}}{3} & \frac{a_{2}}{3} \\
\frac{a_{3}}{3} & \frac{a_{3}}{3} & 1-\frac{2 a_{3}}{3}
\end{array}\right), \quad B=\left(\begin{array}{l}
0 \\
1 \\
0
\end{array}\right) .
$$

One can easily notice that matrix $A$ is stochastic.

The initial condition for the state variable is $x(0)=\left(x_{1}^{0}, x_{2}^{0}, x_{3}^{0}\right)^{\prime}$. The player affects the opinion of only the second agent. The player's aim is to control the opinion of the second agent in such a way as to influence the opinion of two other agents through agent 2 and make their opinions closer to the given value $\hat{x}$ minimizing the costs which implies the minimization problem:

$$
J(u)=\sum_{t=0}^{\infty} \delta^{t}\left[\sum_{i=1}^{3}\left(x_{i}(t)-\hat{x}\right)^{2}+\gamma u^{2}(t)\right]
$$


subject to the state dynamics (2) with initial condition $x(0)=\left(x_{1}^{0}, x_{2}^{0}, x_{3}^{0}\right)^{\prime}$. We rewrite minimization problem in a vector form:

$$
J(u)=\sum_{t=0}^{\infty} \delta^{t}\left[x^{\prime}(t) Q x(t)+q x(t)+3 \hat{x}^{2}+\gamma u^{2}(t)\right]
$$

where $Q=\mathbb{I}_{3}$ is an identity matrix of size three and $q=(-2 \hat{x},-2 \hat{x},-2 \hat{x})$. We should notice that the matrix $Q$ is symmetric and positive definite. The optimization problem is linear-quadratic.

In dynamic games the information structure should be specified when players design their strategies. In the following, we assume that the information structure is feedback Başar and Olsder, 1998, i.e. the player takes into account time and state which can be observed. The feedback strategy of the player is $\psi(t, x)=u \in U$. In the following, we will write $u(t)$ which is the control variable given by feedback strategy $\psi(t, x)$ at time $t$.

We use dynamic programming method. The Bellman equation for the minimization problem takes the form:

$$
V(t, x)=\min _{u(t) \in U}\left[\sum_{i=1}^{3}\left(x_{i}(t)-\hat{x}\right)^{2}+\gamma u^{2}(t)+\delta V(t+1, x(t+1))\right]
$$

where $x(t+1)$ satisfies equation (2). We can rewrite Bellman equation (4) as

$$
V(t, x)=\min _{u(t) \in U}\left[x^{\prime}(t) Q x(t)+q x(t)+3 \hat{x}^{2}+\gamma u^{2}(t)+\delta V(t+1, x(t+1))\right]
$$

Assuming that the value function associated with the minimization problem of (3) be defined as

$$
V(t, x(t))=\sum_{i=1}^{3} k_{i} x_{i}(t)+\sum_{i=1}^{3} \sum_{j=1}^{3} k_{i j} x_{i}(t) x_{j}(t)+k_{0},
$$

where $k_{i j}=k_{j i}, i \neq j$, and $k_{0} \in \mathbb{R}^{1}$. Using the following notations:

$$
K=\left(\begin{array}{lll}
k_{11} & k_{12} & k_{13} \\
k_{21} & k_{22} & k_{23} \\
k_{31} & k_{32} & k_{33}
\end{array}\right), \quad k=\left(k_{1}, k_{2}, k_{3}\right)
$$

we can rewrite the value function as

$$
V(t, x(t))=x^{\prime}(t) K x(t)+k x(t)+k_{0} .
$$

We assume the player uses feedback strategies of the form

$$
u(t, x(t))=c x(t)+c_{0},
$$

where $c=\left(c_{1}, c_{2}, c_{3}\right) \in \mathbb{R}^{3}, c_{0} \in \mathbb{R}^{1}$.

Substituting the expression of $V(t+1, x(t+1))$ from (6) and $x(t+1)$ from (2), we obtain the dynamic programming equation given by 


$$
\begin{aligned}
V(t, x) & =\min _{u(t) \in U}\left[x^{\prime}(t) Q x(t)+q x(t)+3 \hat{x}^{2}+\gamma u^{2}(t)\right. \\
& \left.+\delta x^{\prime}(t+1) K x(t+1)+\delta k x(t+1)+\delta k_{0}\right] \\
& =\min _{u(t) \in U}\left[x^{\prime}(t) Q x(t)+q x(t)+3 \hat{x}^{2}+\gamma u^{2}(t)\right. \\
& \left.+\delta(A x(t)+B u(t))^{\prime} K(A x(t)+B u(t))+\delta k(A x(t)+B u(t))+\delta k_{0}\right]
\end{aligned}
$$

Solving minimization problem in the right-hand side, it implies

$$
u^{*}(t)=-\left(\gamma+\delta B^{\prime} K B\right)^{-1} \delta\left[\frac{1}{2} k B+B^{\prime} K A x(t)\right] .
$$

Taking into account the linear form of control variable (7), we obtain the expressions of coefficients $c_{i}, i=0,1, \ldots, 3$ in the following form:

$$
\begin{aligned}
c_{0} & =-\frac{1}{2} \delta k B\left(\gamma+\delta B^{\prime} K B\right)^{-1}, \\
c & =-\left(\gamma+\delta B^{\prime} K B\right)^{-1} \delta B^{\prime} K A .
\end{aligned}
$$

To find vector $k$ and matrix $K$ we substitute expressions of $u$ and Bellman function into (5) and obtain the following system:

$$
\begin{aligned}
x^{\prime}(t) K x(t)+k x(t)+k_{0} & =x^{\prime}(t) Q x(t)+q x(t)+3 \hat{x}^{2}+\gamma\left(c x(t)+c_{0}\right)^{2} \\
& +\delta\left[(A+B c) x(t)+B c_{0}\right]^{\prime} K\left[(A+B c) x(t)+B c_{0}\right] \\
& +\delta k\left[(A+B c) x(t)+B c_{0}\right]+\delta k_{0} .
\end{aligned}
$$

The vector $k$, matrix $K$ and constant $k_{0}$ can be found as the solution of the following system:

$$
\begin{aligned}
K & =Q+\gamma c^{\prime} c+\delta(A+B c)^{\prime} K(A+B c), \\
k & =q+2 \gamma c c_{0}+\delta k(A+B c)+2 \delta c_{0} B^{\prime} K(A+B c), \\
k_{0} & =3 \hat{x}^{2}+\gamma c_{0}^{2}+\delta c_{0} k B+\delta c_{0}^{2}(B)^{\prime} K B+\delta k_{0} .
\end{aligned}
$$

Therefore, the optimal control minimizing (3) with state dynamics (2) is defined by (8) and solution of the system of equations (11)-(13).

\section{Model with Communication Structure Given by an Incomplete Graph}

Consider a scenario where one connection between agents (between agents 1 and 3 ) is missing. The player controls the opinion of the second agent as in the previous model and his task is to make the opinion of the agents closer to the target opinion of $\hat{x}$. The communication graph is presented in Fig. 2 . 


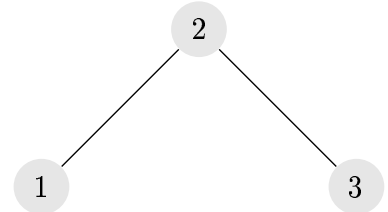

Fig. 2. Communication structure represented by an incomplete graph.

The opinion dynamics in this case is given by the system

$$
\begin{aligned}
& x_{1}(t+1)=x_{1}(t)+a_{1}\left(\frac{x_{1}(t)+x_{2}(t)}{2}-x_{1}(t)\right) \\
& x_{2}(t+1)=x_{2}(t)+a_{2}\left(\frac{x_{1}(t)+x_{2}(t)+x_{3}(t)}{3}-x_{2}(t)\right)+u(t), \\
& x_{3}(t+1)=x_{3}(t)+a_{3}\left(\frac{x_{2}(t)+x_{3}(t)}{2}-x_{3}(t)\right)
\end{aligned}
$$

with initial condition $x(0)=\left(x_{1}^{0}, x_{2}^{0}, x_{3}^{0}\right)$, or in vector form:

$$
x(t+1)=A_{1} x(t)+B u(t)
$$

where $x(t)=\left(x_{1}(t), x_{2}(t), x_{3}(t)\right)^{\prime} \in \mathbb{R}^{3}$, and

$$
A_{1}=\left(\begin{array}{ccc}
1-\frac{a_{1}}{2} & \frac{a_{1}}{2} & 0 \\
\frac{a_{2}}{3} & 1-\frac{2 a_{2}}{3} & \frac{a_{2}}{3} \\
0 & \frac{a_{3}}{2} & 1-\frac{a_{3}}{2}
\end{array}\right)
$$

Making the similar calculations as in Section 3, we obtain

$$
u^{*}(t)=-\left(\gamma+\delta B^{\prime} K B\right)^{-1} \delta\left[\frac{1}{2} k B+B^{\prime} K A_{1} x(t)\right] .
$$

Taking into account the linear form of control variable (7), we obtain the expressions of coefficients $c_{i}, i=0,1, \ldots, 3$ in the following form:

$$
\begin{aligned}
c_{0} & =-\frac{1}{2} \delta k B\left(\gamma+\delta B^{\prime} K B\right)^{-1}, \\
c & =-\left(\gamma+\delta B^{\prime} K B\right)^{-1} \delta B^{\prime} K A_{1},
\end{aligned}
$$

where vector $k$ and matrix $K$ satisfy the following system:

Substituting the expressions of $u$ and Bellman function $V(t, x(t))$ and obtain the following system:

$$
\begin{aligned}
x^{\prime}(t) K x(t)+k x(t)+k_{0} & =x^{\prime}(t) Q x(t)+q x(t)+\hat{x}^{2}+\gamma\left(c x(t)+c_{0}\right)^{2} \\
& +\delta\left[\left(A_{1}+B c\right) x(t)+B c_{0}\right]^{\prime} K\left[\left(A_{1}+B c\right) x(t)+B c_{0}\right] \\
& +\delta k\left[\left(A_{1}+B c\right) x(t)+B c_{0}\right]+\delta k_{0} .
\end{aligned}
$$

The vector $k$, matrix $K$ and constant $k_{0}$ can be found as the solution of the following system:

$$
\begin{aligned}
K & =Q+\gamma c^{\prime} c+\delta\left(A_{1}+B c\right)^{\prime} K\left(A_{1}+B c\right), \\
k & =q+2 \gamma c c_{0}+\delta k\left(A_{1}+B c\right)+2 \delta c_{0} B^{\prime} K\left(A_{1}+B c\right), \\
k_{0} & =3 \hat{x}^{2}+\gamma c_{0}^{2}+\delta c_{0} k B+\delta c_{0}^{2}(B)^{\prime} K B+\delta k_{0} .
\end{aligned}
$$


where vector $c$ and constant $c_{0}$ satisfy equations (17) and (16) respectively.

Therefore, the optimal control minimizing (3) with state dynamics (14) is defined by (15) and solution of the system of equations (18)-(20).

\section{Numerical Simulation}

Using Wolfram Mathematica, we obtain numerical results that allow to analyze and evaluate the convergence of the opinions of agents, as well as the relationship of the remaining parameters of the model with each other. We assume that the initial data of the first and third agents are equal, as well as their coefficients $a_{1}=a_{3}$.

We consider two examples, with complete communication graph and then with incomplete graph.

For the case of complete communication graph, let the parameters of the model be

$$
\begin{aligned}
x_{1}^{0} & =x_{3}^{0}=0.4, x_{2}^{0}=0.7, \\
a_{1} & =a_{3}=0.9, a_{2}=0.8, \\
d & =0.6, \gamma=0.1, \hat{x}=1 .
\end{aligned}
$$

Solving system (2), taking into account expressions (3), (4) and (7), the values of the opinions of all participants are obtained, and the optimal control trajectory is determined. The results for the first 20 periods are presented in Table 1.

\begin{tabular}{c|c|c|c|c|c|c|c|c|c|c|c|c} 
& $t=0$ & $t=2$ & $t=4$ & $t=6$ & $t=8$ & $t=10$ & $t=12$ & $t=14$ & $t=16$ & $t=18$ & $t=20$ \\
\hline$x_{1}(t)$ & 0.400 & 0.578 & 0.688 & 0.743 & 0.771 & 0.786 & 0.793 & 0.797 & 0.799 & 0.800 & 0.800 \\
\hline$x_{2}(t)$ & 0.700 & 0.791 & 0.796 & 0.798 & 0.799 & 0.800 & 0.800 & 0.800 & 0.801 & 0.801 & 0.801 \\
\hline$x_{3}(t)$ & 0.400 & 0.578 & 0.688 & 0.743 & 0.771 & 0.786 & 0.793 & 0.797 & 0.799 & 0.800 & 0.800 \\
\hline$u(t)$ & 0.245 & 0.116 & 0.059 & 0.04 & 0.030 & 0.015 & 0.008 & 0.004 & 0.001 & 0.0005 & 0.0002
\end{tabular}

Table 1. The dynamics of the optimal control and opinions with the one player and three agents for a numerical simulations with parameters (21) and complete communication graph.

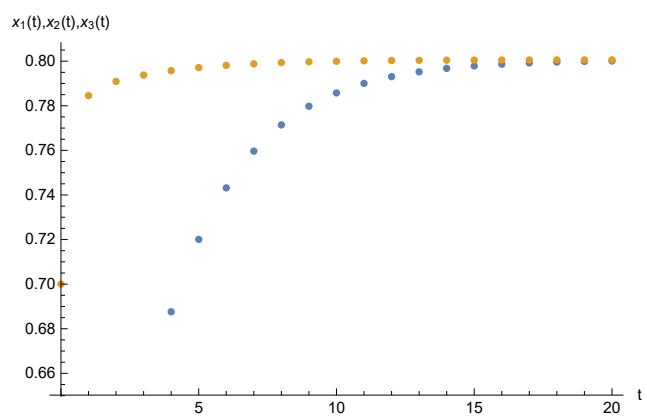

Fig. 3. The dynamics of opinions for three-agent model with complete communication graph and parameters given by (21) (blue - agents 1 and 3, orange - agent 2).

Analyzing the data in Table 1, we conclude that the opinions of the agents, starting from the moment $t=13$, are as close as possible to each other, despite the 
fact that the initial data are different. This fact can be explained by the fact that the individual coefficients of agents 1 and 3 are greater than the coefficient of agent 2 , and the initial opinion is larger for agent 2.

The opinions are in some sense "stabilized" over time. The player controls the opinions of the agents. As the opinions of the agents converge, the optimal control tends to zero, demonstrating a decreasing form of a function. Graphs of agents' opinions are presented in Fig. 3. The optimal control trajectory is represented in Fig. 4.

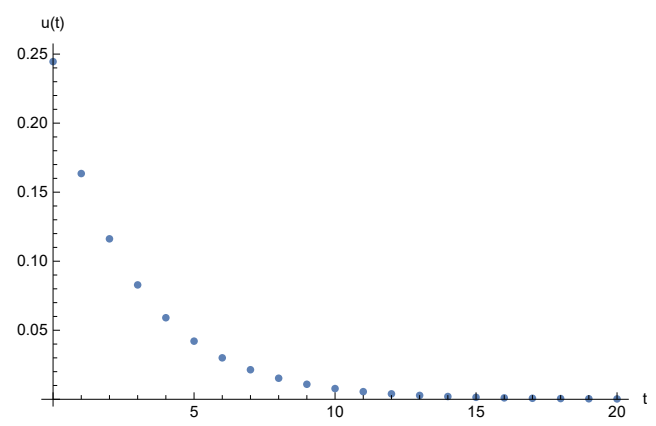

Fig. 4. The dynamics of optimal control for three-agent model with complete communication graph and parameters given by (21).

Now we consider the second example and compare the models with complete and incomplete communication structure. We use the following parameters for modeling:

$$
\begin{aligned}
x_{1}^{0} & =x_{3}^{0}=0.4, x_{2}^{0}=0.5, \\
a_{1} & =a_{3}=0.2, a_{2}=0.5, \\
d & =0.6, \gamma=0.5, \hat{x}=0.6 .
\end{aligned}
$$

Solving systems (2) and (14), taking into account expressions (3), (4), (7) and (15), we obtain the solution including the agents' opinions and optimal control trajectory. In contrast to the previous example, the solution was analyzed for more than fifty time periods. The results are presented in Table 2 .

\begin{tabular}{c|c|c|c|c|c|c} 
& $t=0$ & $t=10$ & $t=20$ & $t=30$ & $t=40$ & $t=50$ \\
\hline$x_{1}(t)$ & 0.400 & 0.364 & 0.334 & 0.317 & 0.307 & 0.302 \\
\hline$x_{2}(t)$ & 0.500 & 0.306 & 0.301 & 0.299 & 0.297 & 0.296 \\
\hline$x_{3}(t)$ & 0.400 & 0.364 & 0.334 & 0.317 & 0.307 & 0.302 \\
\hline$u(t)$ & -0.107 & -0.020 & -0.011 & -0.006 & -0.004 & -0.002
\end{tabular}

Table 2. The dynamics of the optimal control and opinions for a numerical simulations with parameters (22) and complete communication graph.

The numerical results of calculations of the opinions and optimal controls with the parameters defined by (22) for the model with incomplete graph are presented in Table 3. 


\begin{tabular}{c|c|c|c|c|c|c} 
& $t=0$ & $t=10$ & $t=20$ & $t=30$ & $t=40$ & $t=50$ \\
\hline$x_{1}(t)$ & 0.400 & 0.368 & 0.345 & 0.336 & 0.332 & 0.330 \\
\hline$x_{2}(t)$ & 0.500 & 0.335 & 0.331 & 0.329 & 0.329 & 0.329 \\
\hline$x_{3}(t)$ & 0.400 & 0.368 & 0.345 & 0.336 & 0.332 & 0.330 \\
\hline$u(t)$ & -0.086 & -0.012 & -0.005 & -0.002 & -0.001 & -0.0003
\end{tabular}

Table 3. The dynamics of the optimal control and opinions for a numerical simulations with parameters (22) and incomplete communication graph.

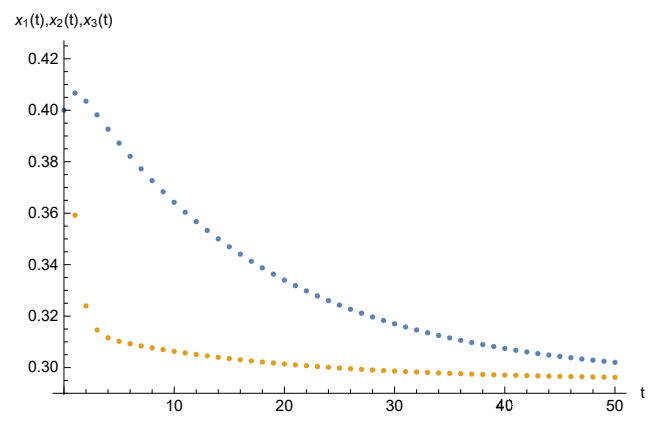

Fig. 5. The dynamics of opinions for three-agent model with complete communication graph and parameters given by (22) (blue - agents 1 and 3, orange - agent 2).

The second example is interesting as the individual coefficient $a_{2}$ of the second agent exceeds the coefficients of agents $a_{1}, a_{3}$ by more than two times. However, despite this fact, the opinions of all three agents have the same decreasing character in time interval $[0 ; 50]$. Opinion dynamics for complete communication graph are presented in Table 2 and Figure 5. The solution of the problem with incomplete communication structure is presented in Table 3 and Figure 6. Contrary to the first example, both models introduce slower changes, although the target opinion $\hat{x}=0.6$ is less than in the first example. The discount factor remains the same in the second example, but the value of $\gamma$ is increased in five times, which can be one of the reasons affecting the nature of the changes.

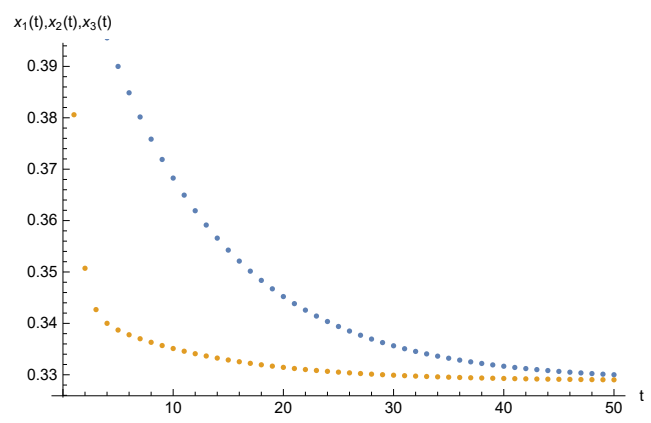

Fig. 6. The dynamics of opinions for three-agent model with incomplete communication graph and parameters given by (22) (blue - agents 1 and 3, orange - agent 2). 
For a model with incomplete communication graph, the convergence of opinions is faster than for complete graph. It can be explained by the following. With incomplete communication structure, agents 1 and 3 are isolated from direct "communication" with each other, and they are influenced directly only by agent 2 whose controlled by the player. This direct communication with the agent 2 and isolation of agents 1 and 3 from each other causes of rapid convergence of opinions.

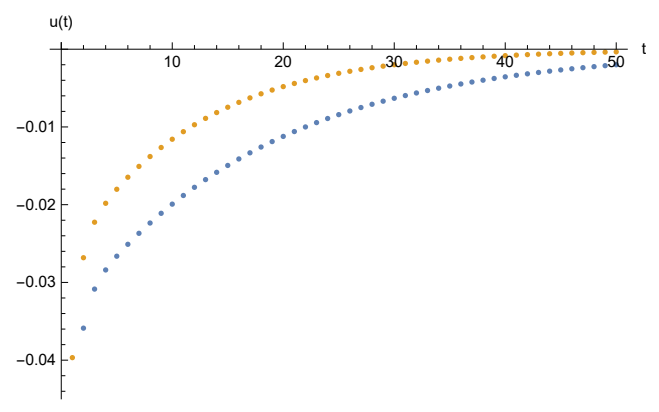

Fig. 7. The dynamics of optimal control for three-agent model with complete (blue) and incomplete (orange) communication graph and parameters given by (22).

Particular attention should be paid to the optimal control dynamics. Both control trajectories (for complete and incomplete structures) are represented in Figure 7. For a model with incomplete graph, the optimal control converges to zero faster than in a model with complete graph. Contrary to the first example, the optimal control for both cases is an increasing function of time. Moreover, the opinions for the scenario with incomplete communication structure over the entire duration are slightly larger than for the scenario with complete communication structure, i.e., closer to the target value.

\section{Conclusion}

The problem of the dynamics of agents' opinions is considered in the paper. The communication structure is defined in the form by a complete or incomplete graph with three nodes representing the agents. The player controls agents' opinions via one agent minimizing his costs on control and the difference between the target and agents' opinions. The minimization problem is solved assuming feedback information structure using dynamic programming method. The control variable influences on the convergence of the agents' opinions to the target one. Therefore, the given dynamics makes the process the so-called "predictable". The numerical value of control decreases in absolute value while the agents' opinions become close to the target opinion. However, the second important factor is the presence and absence of connections between all members of the society. Despite changes in the parameters of the model, incomplete communication structure between agents slows down the convergence of the system.

As an illustration of the theoretical results, we consider examples with different initial opinions of all members of the society, as well as the values of the discount factor, different coefficients in the cost function. 
These parameters affect the rate of the system convergence. We provide the important conclusions about convergence of the system obtained from numerical simulations:

- The higher the price a player pays for control, the slower the process of convergence.

- The process depends on the initial opinions of the agents, i.e., the smaller the difference between them, the faster the convergence.

It is worth noting that we consider the optimization problem with one player, so the problem is not a game. However, this study provides a broad perspective for the further study of conflicting scenarios, in the case when there are more than one player, and we can consider the game as competition on agents' opinions (e.g., see Mazalov and Parilina, 2019).

\section{References}

Başar, T. and Olsder, G. J. (1998). Dynamic noncooperative game theory. New York: Academic Press.

Barabanov, I. N., Korgin, N. A., Novikov, D. A., Chkhartishvili, A. G. (2010). Dynamics models of informational control in social networks. Autom. Remote Control, 71, 24172426 .

Bauso, D., Tembine, H., Başar, T. (2016). Opinion dynamics in social networks through mean field games. Control opinion, 54, 3225-3257.

Bure, V. M., Parilina, E. M., Sedakov A. A. (2017). Consensus in a social network with two principals. Autom. Remote Control, 78, 1489-1499.

Chkhartishvili, A. G., Gubanov, D. A., Novikov, D. A. (2019). Social network: Models of information influence. Control and Confrontation. Switzerland.

De Groot, M. (1974). Reaching a consensus. Am. Stat. Assoc., 69, 119-121.

Dorofeeva, Yu.A. (2020). Management effects on the dynamics of opinions in a team. Proceedings of Karelian Research Centre of RAS, 7, 28-33.

Friedkin, N. and Johnsen, E. (1990). Social influence and opinions. Journal of Mathematical Sociology, 15, 193-206.

Hegselmann, R. and Krause, U. (2005). Opinion dynamics driven by various ways of averaging. Comp. econ., 25, 381-405.

Leonov, G. A. (2004). Theory of control. Saint Petersburg University.

Mazalov, V.V. (2016). Mathematical Game Theory and Applications. Lan Publishing House.

Mazalov, V. and Parilina, E. (2019). Game of competition for opinion with two centers of influence. Mathematical Optimization Theory and Operations Research - 18th International Conference, MOTOR 2019, Proceedings (Lecture Notes in Computer Science (including subseries Lecture Notes in Artificial Intelligence and Lecture Notes in Bioinformatics). Springer, 11548 LNCS, pp. 673-684.

Mazalov, V.V. and Parilina, E. M. (2020). The Euler-Equation Approach in AverageOriented Opinion Dynamics. Mathematics, 8(3), 1-16.

Parilina, E. and Sedakov, A. (2014). Stable Cooperation in Graph-Restricted Games. Contributions to game theory and management, Vol. VII, 271-281.

Rogov, M. A. and Sedakov A. A. (2020). Coordinated Influence on the Opinions of Social Network Members. Automation and Remote Control, 81(3), 528-547.

Sedakov, A. A. and Zhen, M. (2019). Opinion dynamics game in a social network with two influence nodes. Vestn. St. Petersburg Univ. Appl. Math. Comp. Sci., 15, 118-125.

Sirbu, A., Lorento, V., Servedio, V., Francessa, T. (2016). Opinion dynamics: models, extensions and external effects. Physics and Society, 5, 363-401.

Weisbuch, G., Deffuant, G., Amblard, F. (2005). Persuasion dynamics. Physica A, 353, $555-575$. 